Eur. J. Clin. Chem. Clin. Biochem.

Vol. 31,1993 , pp. $23-28$

(C) 1993 Walter de Gruyter \& Co. Berlin - New York

\title{
Pre-Analytical, Analytical and Biological Sources of Variation of Lipoprotein(a)
}

\author{
By M. Panteghini and Franca Pagani
}

\author{
$1^{\circ}$ Laboratorio Analisi Chimico-Cliniche, Spedali Civili, Brescia, Italy
}

(Received June 5/August 17, 1992)

Summary: The pre-analytical, analytical and biological sources of variation were estimated for serum lipoprotein(a) concentrations, as measured with a new commercially available procedure. There were no significant differences between the concentrations of lipoprotein(a) measured in EDTA plasma and serum. The analyte is stable in specimens stored for up to two weeks in the refrigerator, or up to three months at $-20^{\circ} \mathrm{C}$. Fasting is not essential before the lipoprotein(a) determination. Data on analytical (between-run coefficient of variation $<15.7 \%$ ), within-subject $(8.6 \%)$ and between-subject variation $(85.8 \%)$ were used to calculate the analytical goal for imprecision $(<4.3 \%)$, the critical difference between serial values from an individual that represents a significant change (36.3\%), the number of specimens which should be collected to estimate the homeostatic value for an individual to within $\pm 5 \%$ (26), and to establish the marked individuality of lipoprotein(a) (index of individuality, 0.1).

\section{Introduction}

Hyperlipidaemia is now an established major reason for the development of coronary artery disease. Considerable attention has been focussed on levels of low (LDL) and high (HDL) density lipoproteins and the apolipoproteins as risk factor determinants (1). Recently, there has been a considerable resurgence of interest in lipoprotein(a), since there is evidence that the level of circulating lipoprotein(a) represents an independent risk factor for premature coronary artery disease, stroke, and development of atherosclerotic lesions, with possibly greater predictive potential than the other lipoprotein traits (2). The awareness of the striking correlation between atherosclerosis and concentration of lipoprotein(a) in serum prompted the development of several different assays for lipoprotein(a) determination (3). Specific antibodies are now commercially available and lipoprotein(a) can be detected and quantified routinely in any laboratory.

According to Cooper et al. (4), standardization of measurements of lipoproteins requires recognition of the pre-analytical sources of variation, the use of preventive measures to control those variations, and a choice of final conditions that minimize samplerelated sources of variation. Fraser et al. advocated that data on analytical and biological variation, in addition to pre-analytical variability, should be generated early in the evolution of a new test, i.e. lipoprotein(a), to derive the information necessary for assessing desirable performance characteristics or analytical goals (5), the utility of the conventional population-based reference interval (6), and the difference required for a change in serial results in an individual to be significant (7). Furthermore, if a laboratory test is to be used to screen the population, the number of analyses required to assess the homeostatic value for an individual should be determined. The purpose of the present study was to obtain data on these various sources of variation of lipoprotein(a) concentration in patient samples, in order to obtain a complete picture of all the possible components of variability influencing this biochemical test. In particular, definitive information on the biological variation of lipoprotein(a) is so far not available, a fact that may seem surprising when one considers the many reports on serum lipoprotein(a) as a risk factor for premature coronary artery disease and development of atherosclerosis. 


\section{Materials and Methods}

\section{Procedures}

Lipoprotein(a) was determined immunometrically as previously described (8) using the Macra Lp(a) kit assay (Terumo Medical Corp., Elkton, MD 21921, USA). This assay is based on the use of a monoclonal antibody (1D1) raised against the kringle IV domains of apolipoprotein(a) (solid phase), and a polyclonal antibody raised against lipoprotein(a) and conjugated with peroxidase. The minimal detectable lipoprotein(a) concentration was estimated to be $1.5 \mathrm{mg} / \mathrm{l}(8)$. The immunoblotting technique for phenotyping lipoprotein(a) was performed according to Molinari et al. (9). Briefly, after reduction of the serum samples, the apolipoprotein(a) isoforms were separated electrophoretically in a $4-15 \%$ polyacrylamide gel gradient using a commercially available system (PhastSystem, Pharmacia, Uppsala, Sweden). The separated proteins were transferred to nitrocellulose membranes where they were fixed with a polyclonal antihuman lipoprotein(a) serum from sheep (Immuno AG, Wien, Austria). After removal of the unbound proteins by washing and blocking of unreacted sites, an alkaline phosphatase-conjugated rabbit anti-sheep IgG (Chemicon International, Inc., El Segundo, CA 90245, USA) was used as the second antibody. The complex was developed with the substrate (5-bromo-5chloro-3-indoxylphosphate) and the chromogen (nitroblue tetrazolium) to visualize the bands. A lipoprotein(a) phenotyping standard purchased from Immuno AG was run on each gel as a reference.

\section{Effect of anticoagulant}

For preparing plasma specimens for lipoprotein measurement, EDTA is usually the preferred anticoagulant. To investigate the influence of sample type on the lipoprotein(a) concentration, we collected serum and plasma EDTA dipotassium salt (2.5 $\mathrm{mmol} / \mathrm{l})$ specimens from 17 laboratory workers. Lipoprotein(a) was analysed simultaneously in serum or plasma after centrifugation at $4000 \mathrm{~g}$ for 15 minutes at $4{ }^{\circ} \mathrm{C}$.

\section{Effect of storage}

For a preliminary determination of the stability of lipoprotein(a) in serum, a sample containing $200 \mathrm{mg} / \mathrm{l}$ lipoprotein(a) was divided in aliquots of $100 \mu \mathrm{l}$ in capped plastic tubing segments, then kept at $4{ }^{\circ} \mathrm{C}$ and at $-20^{\circ} \mathrm{C}$ without the addition of any preservative. Aliquots were assayed in quadruplicate every other day for two weeks, and subsequently once a week for three months (only for frozen specimen), and the significance of the difference from the original value was tested. Later, to further examine the longer-term effects of storage on lipoprotein(a) isoform pattern, four serum samples with different lipoprotein(a) phenotypes (apo(a) S2, S3, and S4 homozygotes and apo(a) S2/S3 heterozygote according to the nomenclature of Utermann (10)) were divided into aliquots, which were stored at $-20^{\circ} \mathrm{C}$ for one, two, and three months, and assayed after thawing for lipoprotein(a) concentration and isoform pattern. Differences between lipoprotein(a) concentration at different times were analysed by a matched t-test.

\section{Effect of fasting}

Fifteen subjects were studied: 12 females and 3 males, ages $23-73$ years. All were in good health and received no medication. All had serum concentrations of lipoprotein(a) that were above the lower limit of detection of the method. In the morning, after 12 hours fasting, $5 \mathrm{ml}$ blood was drawn from the arm vein of each subject, in order to establish baseline values. Each subject was then given a meal of normal composition ( $40 \%$ fat (polyunsatured/satured, 0.5 ), $45 \%$ carbohydrate, $15 \%$ protein, energy fractions, approximately) and $5 \mathrm{ml}$ blood was drawn at two hours after the beginning of the meal. The blood was centrifuged at $4000 \mathrm{~g}, 4^{\circ} \mathrm{C}$ for 15 minutes, and stored at $-70^{\circ} \mathrm{C}$ until all the samples were available, so that all the analyses were done in the same assay run. . ,

\section{Analytical imprecision}

Within-run imprecision was determined by 20 replicate measurements of three different serum samples with different concentrations of lipoprotein(a) on the same plate in one assay. Between-run imprecision was determined from data on 20 measurements on the same serum samples; between each experiment, the sera were stored as separate samples at $-70^{\circ} \mathrm{C}$.

\section{Biological variability}

The biological variation of lipoprotein(a) was determined by taking five 10-ml blood specimens from each of 8 apparently healthy normolipidaemic laboratory workers (4 men and 4 women, ages 21 to 45 years) once a week for five weeks. The inclusion criteria were that the subjects be within $20 \%$ of ideal body weight, and, for women, have regular menstrual cycles and use a non-hormonal contraceptive method. The design and execution of the experiment were thoroughly explained to the subjects and informed consent was obtained. They were urged not to change their dietary habits or activities, and their weights during the study remained stable within $\pm 1.0 \mathrm{~kg}$. After fasting for 12 to 14 hours and without any morning exercises or smoking, venous blood was obtained in a sitting position for 1 to 5 minutes with minimal stasis by the same phlebotomist between 8 and 9 a.m. Serum specimens, separated by centrifugation $\left(4000 \mathrm{~g}, 15\right.$ minutes), were stored at $-70^{\circ} \mathrm{C}$ until analysed. When all the specimens were available, they were thawed, mixed, and centrifuged to ensure clarity and assayed in a single run in duplicate in random order. To further minimize analytical variation, a single analyst performed all the assays and a single lot of reagents was used.

Biological within-subject variance was estimated from the total within-subject variance minus within-run analytical variance (7). The latter was estimated from the replicate analyses of the specimens from the patients themselves (11). Biological between-subject variance was estimated from the total variance of the set of duplicate data from the assay performed on each subject minus analytical and within-subject components (7). All the components of variation were then transformed to the relevant coefficients of variation using the overall mean (7).

\section{Results and Discussion}

\section{Effect of anticoagulant}

The anticoagulants can cause various degrees of shifts of water from the blood cells into the plasma and thus alter the plasma concentrations of the large particle constituents, like lipoprotein(a).(12). However, serum and plasma EDTA specimens collected from the same subjects gave similar results (median value (and range), $57(7-774)$ vs $58(2-850) \mathrm{mg} / \mathrm{l}$, paired sample Wilcoxon test, $\mathrm{p}=0.74$ ). Thus, lipoprotein(a) can be measured in either plasma EDTA or serum with no significant differences. 
Tab. 1. Serum concentrations and isoform patterns of lipoprotein(a) before and after storage at $-20^{\circ} \mathrm{C}$.

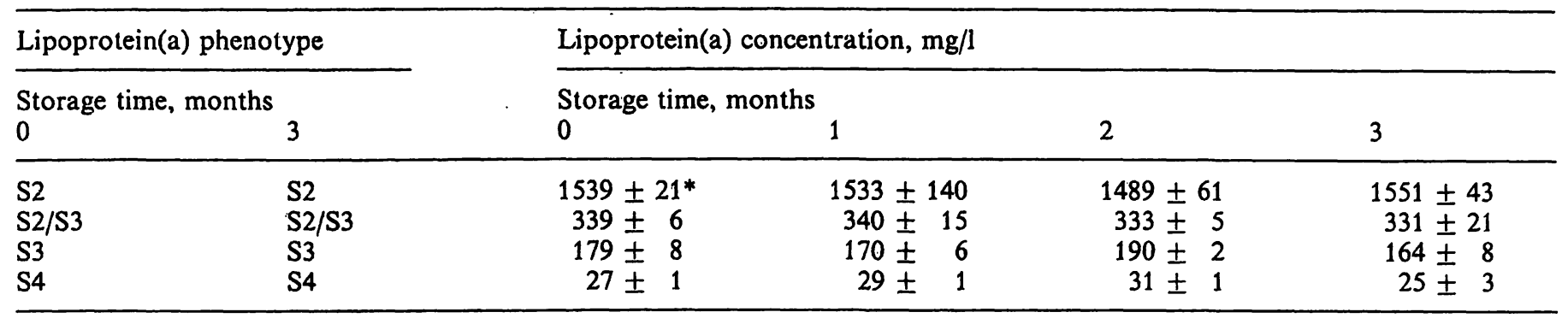

*: mean $\pm S D, n=4$

\section{Effect of storage}

It is known that sample handling and storage may drastically influence the accuracy of the results in many kinds of lipoprotein assay (13). The effect of storage on serum lipoprotein(a) concentration and isoform pattern is showed in figure 1 and table 1 . In agreement with the preliminary results of Fless et al. (14), we observed no significant variations in lipoprotein(a) concentration after serum was stored at $4^{\circ} \mathrm{C}$ for 15 days or at $-20^{\circ} \mathrm{C}$ for three months. Furthermore, we found that serum specimens stored at $-20^{\circ} \mathrm{C}$ for as long as three months were also suitable for lipoprotein(a) phenotyping. Consequently, analyses for lipoprotein(a) should preferably be performed on "fresh" specimens, but when analyses must be delayed, the serum or plasma specimens may be stored (capped) in the refrigerator up to two weeks or at $-20^{\circ} \mathrm{C}$ for a longer period.

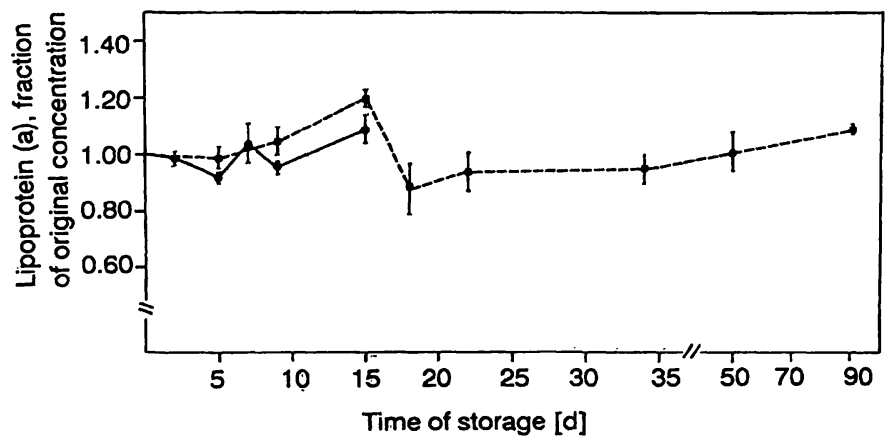

Fig. 1. Stability of lipoprotein(a) concentration in serum when stored at $4^{\circ} \mathrm{C}(\bullet-\bullet)$ and at $-20^{\circ} \mathrm{C}(\square-\cdots)$. Values represent the mean percent recovery \pm SD of four different determinations.

\section{Effect of fasting}

In agreement with recent data by Pfaffinger et al. (15), there were no significant differences between the lipoprotein(a) concentrations of fasting serum and postprandial serum (median value (and range), 86 $(2-418)$ vs $86(2-556) \mathrm{mg} / \mathrm{l}$, paired samples Wilcoxon test, $\mathrm{p}=0.89$ ). Thus, fasting is desirable before a test specimen is collected for a battery of lipids and lipoproteins, but is not essential before lipoprotein(a) is determined.

\section{Analytical imprecision}

Results of imprecision studies are shown in table 2. The coefficients of variations for intra- and interassay imprecision ranged between 5.4 and $15.7 \%$ for the samples we tested.

Tab. 2. Analytical imprecision of lipoprotein(a) determination in patient samples.

\begin{tabular}{|c|c|c|c|c|c|}
\hline \multicolumn{3}{|c|}{$\begin{array}{l}\text { Within-run imprecision } \\
(n=20)\end{array}$} & \multicolumn{3}{|c|}{$\begin{array}{l}\text { Between-run imprecision } \\
(\mathbf{n}=20)\end{array}$} \\
\hline $\begin{array}{l}\bar{x} \\
(\mathrm{mg} / \mathrm{l})\end{array}$ & $\begin{array}{l}\mathrm{SD} \\
(\mathrm{mg} / \mathrm{l})\end{array}$ & $\begin{array}{l}\mathrm{CV} \\
(\%)\end{array}$ & $\begin{array}{l}\bar{x} \\
(m g / l)\end{array}$ & $\begin{array}{l}\mathrm{SD} \\
(\mathrm{mg} / \mathrm{l})\end{array}$ & $\begin{array}{l}\text { CV } \\
(\%)\end{array}$ \\
\hline $\begin{array}{r}95 \\
299 \\
660\end{array}$ & $\begin{array}{l}10 \\
34 \\
36\end{array}$ & $\begin{array}{r}10.5 \\
11.4 \\
5.4\end{array}$ & $\begin{array}{l}108 \\
279 \\
645\end{array}$ & $\begin{array}{l}17 \\
32 \\
47\end{array}$ & $\begin{array}{r}15.7 \\
11.5 \\
7.3\end{array}$ \\
\hline
\end{tabular}

\section{Biological variability}

Table 3 shows the components of biological variation, expressed as coefficients of variation (CV), and certain indices derived from the data on biological variation, namely, the analytical goal, the index of individuality, the critical difference required for serial results from an individual to have changed significantly at $p \leqslant 0.05$, and the number of specimens which should be collected to estimate the homeostatic value for an individual to within $\pm 5 \%$. Generally, serum lipoprotein(a) concentration showed larger intra- and interindividual fluctuations than other lipids and lipoproteins, with the exception of the within-subject biolog-

Tab. 3. Overall mean value, components of biological variation, and derived indices for serum lipoprotein(a) concentration.

Mean of all results

Within-subject variation $\left(\mathrm{CV}_{\mathrm{l}}\right)$

Between-subject variation $\left(\mathrm{CV}_{\mathrm{G}}\right)$

Analytical goal

Index of individuality

Critical difference

Number of specimens required to estimate

the individual's homeostatic value

to within $\pm 5 \%$ 
ical variation of triacylglycerols (reported values, $18.2 \%$ to $21.1 \%)(16,17)$. Unlike the within-subject biological variation, which represents the random fluctuation around an individual's own homeostatic value, the interperson biological variation for lipids and lipoproteins primarily includes differences caused by age, sex, and genetics (4). Population studies of changes in lipoprotein(a) concentration with age and sex indicate that women tend to have higher lipoprotein(a) values than men only after age 45 (18). Therefore, the high value of between-subject variation for lipoprotein(a) is mainly genetically based. On the other hand, the existence of a genetic size polymorphism of apolipoprotein(a), the typical protein component of lipoprotein(a), and of a strong inverse relationship between the apparent molecular mass of apolipoprotein(a) isoforms and plasma concentrations of lipoprotein(a) are well known (19). In view of the large inter-individual differences in lipoprotein(a) concentrations in serum, with values ranging virtually from zero to well over $1000 \mathrm{mg} / \mathrm{l}$, a total of eight individuals, although with a wide range of lipoprotein(a) concentrations ( 7 to $306 \mathrm{mg} / \mathrm{l}$ ), is perhaps too small to be representative of the population at large and could lead to an underestimation of the true value of between-subject variation. However, the value obtained for between-subject variability in our study was already much elevated, i. e., about two- to threefold the published between-subject variability of triacylglycerols (17), thus well expressing the marked heterogeneity of this lipoprotein.

Numerical data on biological variation have important applications. Analytical goals are currently best derived from these data (5). The widely accepted statistical postulate of Harris (20) states that maximum allowable analytical imprecision should be less than or equal to one-half of the within-subject biological variation, i.e., about $4.3 \%$ for lipoprotein(a) from our findings. The validity of this approach is supported by the marked constancy of biological variation data over time, between different sizes of subject group, and between subject groups from a variety of countries (21). Data for analytical imprecision in table 2 show that in practice the analytical goal for lipoprotein(a) is not achieved. Therefore, improvement in the measurement of lipoprotein(a) is required if this assay is to be offered by the routine laboratory.

The ratio of within-subject to between-subject biological variability provides information on the utility of conventional population-based reference intervals (6). If this index of individuality is low, $<0.6$, then the use of reference intervals is of little value and may be misleading. If the index is greater than 1.4, then the reference intervals are of significant utility (22). For lipoprotein(a), this index is 0.1 ; the reference interval is therefore of little use in the interpretation of results of the assay of this analyte. This justifies the attempt to adopt cut-off points based on relative risk of coronary artery disease, although, the setting of such criteria is very difficult $(23,24)$. After all, an approach similar to that of plasma cholesterol is undoubtedly required for lipoprotein(a) and it is considered that the desire to establish a reference interval is a waste of valuable and scarce resources (25).

The results of biological in addition to analytical variation are also used for the critical evaluation of the significance of changes in results obtained from analyses of serial specimens (7). In other words, to interpret serial results objectively it is necessary to know the change which must occur before significance can be claimed. This "critical" difference depends on both analytical and within-subject variability and, at $p \leqslant 0.05$, is $2.77\left(C V_{A}^{2}+C V_{I}^{2}\right)^{1 / 2}$, where $C V_{A}$ is the analytical coefficient of variation and $C V_{I}$ is the within-subject biological coefficient of variation (26). The critical difference for lipoprotein(a) at the cut-off point of $300 \mathrm{mg} / \mathrm{l}$, considered by some authors the best discrimination value for risk of coronary artery disease $(23,24)$, was about $35 \%$. Thus, serial results for lipoprotein(a) for a single subject (if performed with the method in this study) must change more than $35 \%$ before significance can be claimed.

In many clinical studies on lipoprotein(a), only one specimen was collected from each subject, and the analytical results were then used to evaluate the test and investigate the relationships between variables. However, a result from the analysis of a single patient specimen should be interpreted as a lipoprotein(a) concentration lying within certain limits. These limits are dependent on the analytical variation of the method used and also on the intra-individual biological variation, and they can be calculated as the result obtained $\pm 1.96\left(C V_{A}^{2}+C V_{I}^{2}\right)^{1 / 2} \%(95 \%$ confidence limits) (27). Repeated specimens can reduce these limits. In particular, the effective variability falls by the square root of the number of measurements

$$
\left(\frac{1.96\left(\mathrm{CV}_{\mathrm{A}}^{2}+C V_{\mathrm{I}}^{2}\right)^{1 / 2}}{\mathrm{n}^{1 / 2}}\right)
$$

$(6,7)$. From the data obtained in this study, it is therefore possible to estimate the number of specimens required to determine an individual's homeostatic value within, e.g., $\pm 5 \%$. With an average analytical coefficient of variation of $10 \%$, the number of specimens required for lipoprotein(a) is 26 . 
If population screening were introduced, it would be necessary to critically assess the current procedures for the interpretation of results. For example, a result of $330 \mathrm{mg} / 1$ has $95 \%$ confidence limits of $245-415$ $\mathrm{mg} / \mathrm{l}$ and could be either in the high risk (415) or in the low risk category (245) using the recommended cut-off point of $300 \mathrm{mg} / \mathrm{l}$ (28). However, repeated specimen collection and analysis can reduce the confidence limits of the result. In particular, for a mean lipoprotein(a) of $330 \mathrm{mg} / \mathrm{l}$ eight specimens would be required to establish that the individual has a lipoprotein(a) value greater than $300 \mathrm{mg} / \mathrm{l}$. According to Ford (27), this concept can be developed further since there comes a point where the lipoprotein(a) concentration is such that an individual is clearly in the high or in the low risk group. At the $300 \mathrm{mg} / \mathrm{l}$ cut-off point, an observed lipoprotein(a) concentration of greater than $405 \mathrm{mg} / 1$ has $95 \%$ confidence that the true value is greater than $300 \mathrm{mg} / \mathrm{l}$, and an observed concentra-

\section{References}

1. Grundy, S. M. (1990) Cholesterol and Coronary Heart Disease. Scand. J. Clin. Lab. Invest. 50 (suppl. 199), 17-24.

2. Scanu, A. M., Lawn, R. M. \& Berg, K. (1991) Lipoprotein(a) and Atherosclerosis. Ann. Intern. Med. 115, 209218.

3. Albers, J. J., Marcovina, S. M. \& Lodge, M. S. (1990) The Unique Lipoprotein(a): Properties and Immunochemical Measurement. Clin. Chem. 36, 2019-2026.

4. Cooper, G. R., Myers, G. L., Smith, S. J. \& Sampson, E. J. (1988) Standardization of Lipid, Lipoprotein, and Apolipoprotein Measurements. Clin. Chem. 34, B95-B105.

5. Fraser, C. G. (1988) The Application of Theoretical Goals Based on Biological Variation Data in Proficiency Testing. Arch. Pathol. Lab. Med. 112, 404-415.

6. Fraser, C. G. \& Hyltoft Petersen, P. (1991) The Importance of Imprecision. Ann. Clin. Biochem. 28, 207-211.

7. Fraser, C. G. \& Harris, E. K. (1989) Generation and Application of Data on Biological Variation in Clinical Chemistry. C. R. C. Lab. Sci. 27, 409-437.

8. Panteghini, M., Bonora, R., Pagani, F. (1991) A Monoclonal-Antibody-Based Enzyme-Linked Immunosorbent Assay for Determination of Lipoprotein(a) in Serum Evaluated. Giorn. It. Chim. Clin. 16, 361-367.

9. Molinari, E., Pichler, P., Reschny, A. \& Kostner, G. (1990) Lp(a) Phenotyping on the PhastSystem (Pharmacia). Presented at the 55th Annual Meeting of the European Atherosclerosis Society, Brugge, Belgium.

10. Utermann, G. (1990) Genetics of the $L p(a)$ Lipoprotein. In: Lipoprotein (a) (Scanu, A. M., ed.) pp. 75-85, Academic Press, Inc., London.

11. Young, D. S., Harris, E. K. \& Cotlove, E. (1971) Biological and Analytic Components of Variation in Long-Term Studies of Serum Constituents in Normal Subjects. IV. Results of a Study Designed to Eliminate Long-Term Analytic Deviations. Clin. Chem. 17, 403-410.

12. Grande, F., Amatuzio, D. S. \& Wada, S. (1964) Cholesterol Measurement in Serum and in Plasma. Clin. Chem. 10, $619-626$.

13. Bachorik, P. S. (1982) Collection of Blood Samples for Lipoprotein Analysis. Clin. Chem. 28, 1375-1378.

14. Fless, G. M., Snyder, M. L. \& Scanu, A. M. (1989) Enzymelinked Immunoassay for Lp(a). J. Lipid. Res. 30, 651-662. tion of lower than $238 \mathrm{mg} / \mathrm{l}$ has $95 \%$ confidence that the true value is lower than $300 \mathrm{mg} / \mathrm{l}$. It would therefore be acceptable to measure lipoprotein(a) on only one specimen if a value of $>405 \mathrm{mg} / 1$ or $<238 \mathrm{mg} / 1$ is obtained because this can be clearly distinguished from a lipoprotein(a) value of $300 \mathrm{mg} / \mathrm{l}$. However, it should be clear that if population screening for the assessment of risk for coronary artery disease were based on the measurement of lipoprotein(a), it would be necessary to analyse multiple specimens from a significant number of patients, thereby increasing the cost of the screening programme.

\section{Acknowledgement}

We appreciate the excellent technical assistance of Mrs. Carla Bosio. Thanks are also due to Mrs. Olga Alebardi for the lipoprotein(a) phenotype determinations.
15. Pfaffinger, D., Schuelke, J., Kim, C., Fless, G. M. \& Scanu, A. M. (1991) Relationship between apo(a) isoforms and $\mathrm{Lp}$ (a) density in subjects with different apo(a) phenotype: a study before and after a fatty meal. J. Lipid Res. 32, 679-683.

16. Wasenius, A., Stugaard, M., Otterstad, J. E. \& Froyshov, D. (1990) Diurnal and Monthly Intra-Individual Variability of the Concentration of Lipids, Lipoproteins and Apoproteins. Scand. J. Clin. Lab. Invest. 50, 635-642.

17. Ortolà, J., Castineiras, M. J. \& Fuentes-Arderiu, X. (1992) Biological Variation Data Applied to the Selection of Serum Lipid Ratios Used as Risk Markers of Coronary Heart Disease. Clin. Chem. 38, 56-59.

18. Sandkamp, M. \& Assmann, G. (1990) Lipoprotein(a) in PROCAM Participants and Young Myocardial Infarction Survivors. In: Lipoprolein(a) (Scanu, A. M., ed.) pp. 205209, Academic Press, Inc., London.

19. Gaubatz, J. W., Ghanem, K. I., Guevara, J., Nava, M. L., Patsch, W. \& Morrisett, J. D. (1990) Polymorphic Forms of Human Apolipoprotein(a): Inheritance and Relationship of their Molecular Weight to Plasma Levels of Lipoprotein(a). J. Lipid Res. 31, 603-613.

20. Harris, E. K. (1979) Statistical Principles Underlying Analytical Goal-Setting in Clinical Chemistry. Am. J. Clin. Pathol. 72, 374-382.

21. Gowans, E. M. S. \& Fraser, C. G. (1987) Longer-Term Biological Variation of Commonly Analyzed Serum Constituents. Clin. Chem. 33, 717.

22. Harris, E. K. (1974) Effects of Intra- and Inter-Individual Variation on the Appropriate Use of Normal Ranges. Clin. Chem. 30, 1535-1542.

23. Kostner, G. M., Avogaro, P., Cazzolato, G., Marth, E., Bittolo-Bon, G. \& Qunici, G. B. (1981) Lipoprotein Lp(a) and Risk for Myocardial Infarction. Atherosclerosis 38, $51-61$.

24. Armstrong, V. M., Cremer, P., Eberle, E., Manke, A., Schulze, F., Wieland, H., Kreuzer, H. \& Seidel, D. (1986) The Association between Serum Lp(a) Concentrations and Angiographically Assessed Coronary Atherosclerosis. Atherosclerosis $62,249-257$. 
25. The Expert Panel (1988) Report of the National Cholesterol Education Program Expert Panel on Detection, Evaluation, and Treatment of High Blood Cholesterol in Adults. Arch. Intern. Med. 148, 36-69.

26. Fraser, C. G. \& Fogarty, Y. (1989) Interpreting Laboratory Results. Br. Med. J. 298, 1659-1660.
27. Ford, R. P. (1989) Essential Data Derived from Biological Variation for Establishment and Use of Lipid Analyses. Ann. Clin. Biochem. 26, 281-285.

28. Ghilain, J. M., Parfonry, A., Kozyreff, V. \& Heller, F. R. (1988) Lipoprotein(a), Cholesterol, and Coronary Heart Diseases. The Lancet $i i, 963$.

Dr. Mauro Panteghini

$1^{\circ}$ Laboratorio Analisi Chimico-Cliniche Spedali Civili

I-25123 Brescia

Italy 